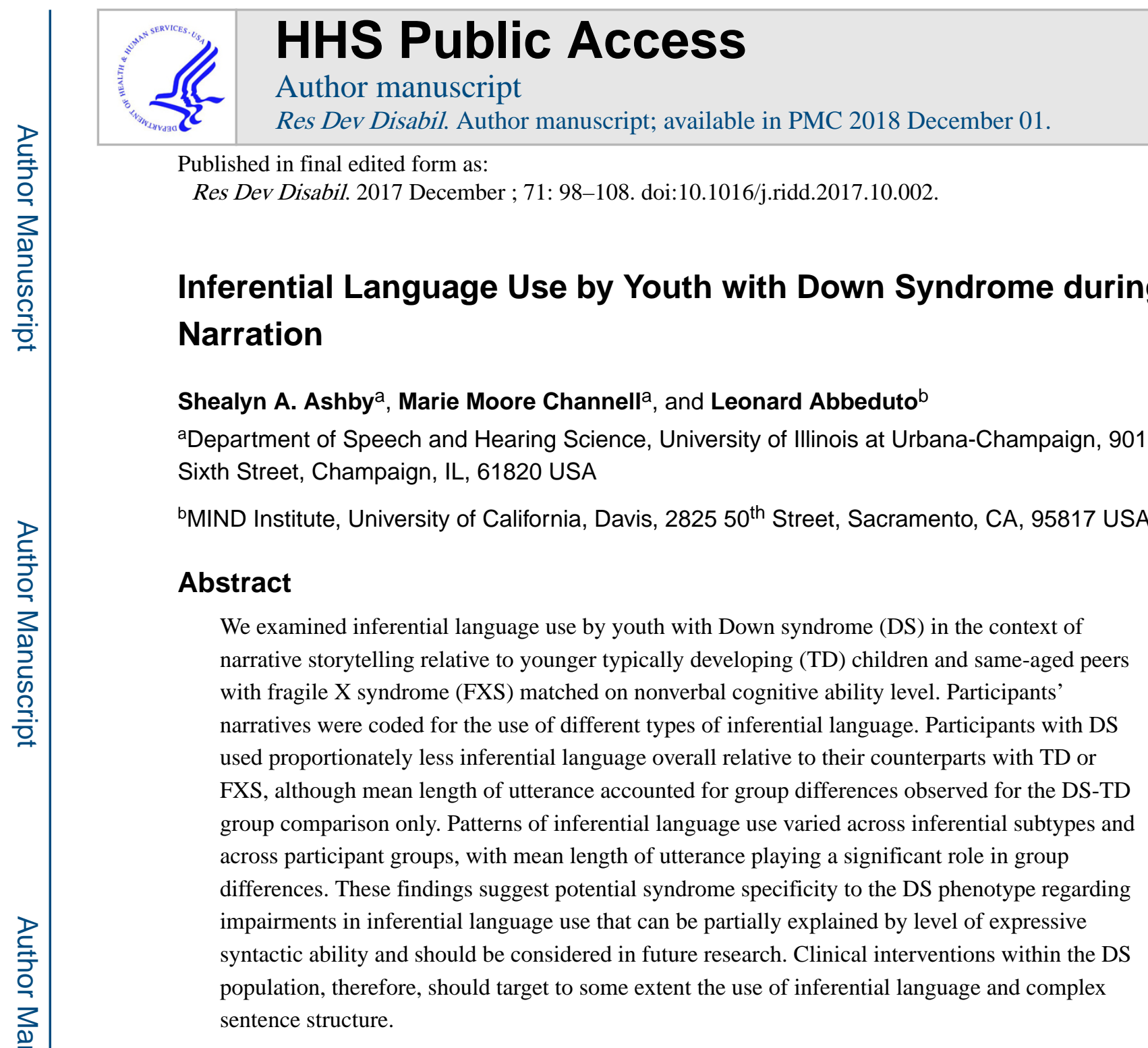

\title{
Keywords
}

inferential language; Down syndrome; intellectual disability; fragile X syndrome; narrative language; social cognition

Down syndrome (DS) is the leading known genetic cause of intellectual disability (Presson et al., 2013). In addition to their cognitive delay, individuals with DS experience difficulties with nearly all facets of language (Abbeduto, Warren, \& Conners, 2007; McDuffie,

Thurman, Channell, \& Abbeduto, 2017). Expressive language is particularly impaired, with delays observed relative to both receptive language and nonverbal cognitive ability level (Chapman, Seung, Schwartz, \& Kay-Raining Bird, 1998; Miller, 1999). Syntax, or grammar, is also particularly impaired relative to other domains of language, such as vocabulary

Correspondence concerning this article should be addressed to: Marie Channell, Department of Speech and Hearing Science, University of Illinois at Urbana-Champaign, 901 S. Sixth Street, Champaign, IL 61820., channell@illinois.edu, Tel: 217-300-3613.

Publisher's Disclaimer: This is a PDF file of an unedited manuscript that has been accepted for publication. As a service to our customers we are providing this early version of the manuscript. The manuscript will undergo copyediting, typesetting, and review of the resulting proof before it is published in its final citable form. Please note that during the production process errors may be discovered which could affect the content, and all legal disclaimers that apply to the journal pertain. 
(Abbeduto et al., 2003; Finestack, Sterling, \& Abbeduto, 2013; Phillips, Loveall, Channell, \& Conners, 2014), with expressive morphosyntax being the most impaired (Chapman \& Hesketh, 2000; Finestack \& Abbeduto, 2010). Far less is known about the pragmatic language abilities, or social use of language, of individuals with DS, particularly for individuals who have progressed beyond pre-linguistic babbling or single-word use. The limited literature points to a complex pragmatic language profile of relative strengths and difficulties, varying by sub-domain assessed and comparison group (e.g., autism spectrum disorder and fragile X syndrome; typical development) used (Abbeduto et al., 2008; Lee et al., 2017; Martin, Losh, Estigarribia, Sideris, \& Roberts, 2013; Roberts et al., 2007; Smith, Naess, \& Jarrold, 2017).

Inferential language is a complex sub-domain of pragmatic language that requires an individual to use social cognition to integrate information that is not explicitly provided from the context, draw logical conclusions (i.e., generate inferences), and use language to communicate these inferences to a listener. To date, no studies have comprehensively examined inferential language abilities in individuals with DS. Because inferential language serves as a framework for positive social interactions and learning in academic contexts (Carpendale \& Lewis, 2006; Symons, 2004), it is important to determine whether the phenotypic characteristics of DS impact this important foundation for development. The purpose of the current study, therefore, was to investigate inferential language use by youth with DS in the context of narrative storytelling.

\section{Inferential Language in Narrative}

Narrative storytelling (i.e., the ability to coherently relay a sequence of events to a listener) is an important aspect of expressive language needed for everyday communication and is an area in which individuals with DS also struggle, although the extent of their challenges in narration is not clear (Boudreau \& Chapman, 2000; Channell, McDuffie, Bullard, \& Abbeduto, 2015; Finestack, Palmer, \& Abbeduto, 2012; Hesketh \& Chapman, 1998; HoganBrown, Losh, Martin, \& Mueffelmann, 2013; Miles \& Chapman, 2002). Narrative storytelling is a functional skill and a means for communicating personal experiences or fictional stories to others, often serving as a forum for reflecting on past events and discussing one's own and others' perspectives (Aldrich, Tenenbaum, Brooks, Harrison, \& Sines, 2011; Bamberg \& Marchman, 1990; Berman, 1995; Trabasso, Stein, Rodkin, Munger, $\&$ Baughn, 1992). Thus, narrative storytelling requires the coordination of abilities across the cognitive, linguistic, and social cognitive domains. For example, an individual must recall previous events and communicate the most relevant, salient information to a listener in an orderly, coherent fashion (McCabe \& Peterson, 1990). Coupled with its functional importance, this makes narrative an ideal context for providing insight into many aspects of the language phenotype associated with DS, including inferential language abilities.

Inferential language is a critical aspect of narrative storytelling because it involves conveying details about events (e.g., cause and effect) and character perspectives (e.g., intentions, actions, and reactions) in a story. In other words, inferential language provides explanations for why events happened, why characters acted in a certain way, how the characters felt, etc. Inferential language also provides other evaluative and descriptive language that naturally 
occurs during narrative storytelling. Inferential language enriches stories and engages the social partner, thus acting as a framework for positive social interaction. It also provides opportunities to discuss others' perspectives and express empathy (Symons, 2004), underscoring its importance to positive peer relationships and friendships (Carpendale \& Lewis, 2006). In sum, inferential language is an important foundation for both narrative and social development.

In typical development, children are able to make inferences in the context of narrative by age 4 (Kendeou, Bohn-Gettler, White, \& van den Broek, 2008; Tompkins, Guo, \& Justice, 2013; Wenner, 2004), and in addition to its crucial role in social communicative competence, this skill is linked to receptive and expressive language abilities as well as later academic achievement (Kendeou et al., 2008; Tompkins et al., 2013). Academically, inferential language is important because the ability to draw inferences from stories is a strong predictor of later developing reading comprehension (Cain \& Oakhill, 1999; 2012). Despite its functional importance and role in later skill development, inferential language use has not yet been fully examined in DS. Although inferential language is discussed above as a pragmatic language skill, its use also requires the coordination of semantic knowledge, cognition, and expressive language abilities, among others. The phenotypic pattern of expressive language and narrative storytelling difficulties along with both strengths and weaknesses in social communication in DS makes it difficult to predict inferential language ability in this population. A better understanding of the development of this skill in DS will provide insight into how interventions may support narrative and social competence in individuals with DS.

\section{Inferential Language in DS}

What is currently known about inferential language use in DS comes from a handful studies focusing on mental state language and causal referencing in autism spectrum disorder in which DS was included as a comparison group (Baron-Cohen, Leslie, \& Frith, 1986; TagerFlusberg, 1992). Although both the Baron-Cohen and Tager-Flusberg studies included very small sample sizes, they pointed to a general trend of young children with DS making fewer references to character mental states (Baron-Cohen et al., 1986) and causality (Baron-Cohen et al., 1986; Tager-Flusberg, 1992), though this deficit depended on the communication context (i.e., spontaneous speech during free play vs. prompted narratives of simple picture sequences).

Additionally, two studies have examined a related skill —-the use of evaluations-from narratives produced by individuals with DS in their narrations of wordless picture books (Hogan-Brown et al., 2013; Keller-Bell \& Abbeduto, 2007). Evaluations refer to content that enriches the story, such as character dialogue, mental state verbs, sound effects, exaggerations, and repetition. Evaluations can include inferential language (e.g., mental state language) but also repetition used for emphasis and clarity. Evaluations can also include personal opinions to emphasize one's own perspective of the story, whereas inferential language is more focused on inferring others' perspectives. 
Hogan-Brown et al. (2013) examined evaluations without distinguishing between different types of evaluation holistically (i.e., causal references, character emotions and cognitions, intensifiers and attention-getters, and other storytelling devices not specified) and did not find significant group differences among youth with DS, age-matched youth with fragile $\mathrm{X}$ syndrome (FXS; another genetic disorder associated with intellectual disability), or younger typically developing (TD) children matched on an expressive-receptive language (i.e., Expressive Vocabulary Test/Peabody Picture Vocabulary Test-III) composite in their overall use of evaluations, though subtype scores were not reported. In contrast, Keller-Bell and Abbeduto (2007) found that adolescents and young adults with DS included a greater proportion of evaluations in their narratives than peers with FXS or TD matched by nonverbal mental age. A closer examination across the six different types of evaluations included in Keller-Bell and Abbeduto's analysis (mental state verbs, character names, character dialogue, repetition, sound effects, and exaggeration) revealed that this group difference was likely driven by the group with DS using significantly more sound effects than the other groups. These two studies provide initial evidence that the use of evaluations within narrative storytelling may not be impaired in individuals with DS, at least relative to their general developmental delay. However, both studies included some evaluation types that could be considered inferential in nature (e.g., character dialogue; mental state verbs) as well as content that may not be inferential (e.g., exaggeration; repetition).

Based on the studies to date, it is not clear whether inferential language use is a relative strength or weakness in individuals with DS. It is reasonable to hypothesize that performance depends on the type of inference, with those requiring higher-level social reasoning or more complex linguistic structures to communicate the inference being more impaired. The literature on social cognition in DS supports this hypothesis, with several studies documenting early emerging social strengths (Fidler, 2006; Fidler, Most, BoothLaForce, \& Kelly, 2008; Moore, Oates, Hobson, \& Goodwin, 2002), but deficits in higherorder social cognitive reasoning, such as theory of mind (Abbeduto et al., 2001; Zelazo, Burack, Benedetto, \& Frye, 1996), interpreting others' internal states (Beeghly \& Cicchetti, 1997; Hesketh \& Chapman, 1998), and understanding the intentions of others (Hahn, Fidler, Hepburn, \& Rogers, 2013). It is likely, therefore, that these deficits will have an impact on, as well as be impacted by, at least some aspects of inference generation in DS (see TagerFlusberg, 1992). However, this hypothesis has not yet been directly tested, and a more comprehensive examination of inferential language use in individuals with DS is needed.

In contrast, a relatively large body of research has examined the pragmatic language profile of individuals with FXS, suggesting patterns of impairment contextualized within the broader phenotype of FXS (Abbeduto et al., 2008; Estigarribia et al., 2011; Klusek, Martin, \& Losh, 2014; Losh, Martin, Klusek, Hogan-Brown, \& Sideris, 2012; Martin et al., 2012; Roberts et al., 2007; Simon, Keenan, Pennington, Taylor, \& Hagerman, 2001; Sudhalter \& Belser, 2001). Thus, as further explained below, individuals with FXS who also have intellectual disability provide an interesting comparison group for considering inferential language abilities in DS. 


\section{Current Study}

The purpose of the current study was to examine inferential language use in the context of narrative storytelling by individuals with DS. We analyzed the use of different types of inferences that vary in complexity of social-cognitive reasoning to determine patterns of strength and weakness across the inference types. We included two comparison groups: younger TD children and same-age peers with FXS, with the groups matched on nonverbal cognitive ability level. We compared participants with DS to younger TD children of similar nonverbal cognitive ability level to determine whether inferential language is a strength or weakness relative to cognitive developmental level. Including a same-age comparison group of FXS - another cause of intellectual disability - in addition to the TD group provided some insight into the syndrome-specificity of the findings (i.e., the extent to which any differences observed can be attributed to intellectual disability in general or specific phenotypes—DS or FXS—within intellectual disability). Furthermore, the same-age comparisons eliminated any potential group differences that could be attributed to chronological age, a proxy for general life experiences.

Finally, we also examined whether potential group differences remain after accounting for expressive morphosyntax ability (i.e., MLU), because it is possible that individuals with DS do not have the structural language (e.g., syntactic complexity) to communicate narrative content as effectively as TD children (e.g., evaluations; story theme components; Boudreau \& Chapman, 2000; Finestack \& Abbeduto, 2010; Keller-Bell \& Abbeduto, 2007; Miles \& Chapman, 2002). Furthermore, there is some evidence linking structural language to broader pragmatic language skill development in children and adolescents with DS (Lee et al., 2017; Tager-Flusberg \& Anderson, 1991), emphasizing the need to account for language in the current study. Given that expressive morphosyntax, in particular, is a phenotypic weakness in DS, we accounted for the potential role of MLU in our models examining group differences.

In summary, the aims of the current study were to: (1) compare the total amount of inferential language used by individuals with DS relative to those with TD or FXS; (2) determine whether there were group differences in use by inference type; and (3) determine whether potential group differences remain after accounting for MLU.

\section{Method}

\section{Participants}

The 68 participants for this study (DS $n=23, \mathrm{FXS} n=22$, TD $n=23$ ) were drawn from a larger longitudinal project focusing on language development in neurodevelopmental disorders. Participants overlapped with those reported in previous papers (e.g., Channell et al. 2015; Finestack et al., 2013; Kover, McDuffie, Abbeduto, \& Brown, 2012; McDuffie et al., 2010); however, all analyses in the present study are unique and have not been previously reported.

In the larger project, all participants were required to be native English speakers and pass a hearing screening with a pure-tone threshold of $<30 \mathrm{~dB}$ in at least one ear. Participants with 
DS or FXS were included if parent reports indicated their primary means of communication was speech, they were able to produce at least three-word phrases in daily communication, and they had no severe uncorrected sensory or motor impairments that would interfere with participation. A diagnosis of DS was confirmed via documentation from the participant's physician or from parent report when such documentation was not available; documentation of genetic testing was required to confirm a full mutation of the FMR1 gene for all participants with FXS. Finally, TD participants were included if they met the aforementioned criteria and were not receiving special education or speech-language services.

Participants who met these eligibility criteria were included in the current study if they also completed the Narrative Task by providing speech relevant to the story on at least $75 \%$ of the book pages. All participants with DS who met this additional criterion were included, resulting in a final sample size of 23 individuals ( 13 males, 10 females), with ages between 10 and 16 years. We then used Leiter-R (Roid \& Miller, 1997) nonverbal cognitive growth score values to match the participants with DS to same-aged youth with FXS (19 males, 3 females; $t(43)=-0.332, p=.742)$ and 23 younger TD children (14 males, 9 females; $t(44)=$ $-0.058, p=.954)$. The participants with FXS and TD were also matched on Leiter-R growth scores, $t(43)=-0.274, p=.785$. See Table 1 for participant characteristics.

\section{Measures}

Narrative Task-The narrative language sampling procedure developed by Abbeduto and colleagues (e.g., Abbeduto, Benson, Short, \& Dolsih, 1995; Berry-Kravis et al., 2013; Kover et al., 2012) was used to assess participants' abilities to tell narratives from wordless picture books. Participants viewed one of two books, Frog Goes to Dinner or Frog on His Own (counterbalanced across participants). During the exposure period, participants previewed the book, page by page, with an examiner and were instructed to look at each page carefully to see what was happening in the story. Examiners controlled the page turns to standardize the amount of exposure to each page spread (i.e., approximately 10 seconds). Next, participants were prompted to tell the story to the examiner as they viewed the book a second time. Examiner prompts were minimal and scripted to ensure standardization across participants and limit scaffolding. The examiner again controlled the page turns, giving participants approximately 5 to 7 seconds after their last utterance before turning the page. Each participant's narrative was audio-recorded for later transcription, and examiners stated "next page" during each page turn to assist transcribers in tracking location within the book.

Transcription: Trained transcribers used Systematic Analysis of Language Transcripts (SALT; Miller \& Iglesias, 2006) software to transcribe the participants' audio-recorded narratives into appropriately rendered text files. Participants' utterances were segmented into communication units (C-units; Loban, 1976), which contain an independent clause along with its modifiers that include dependent clauses, and notated according to standard SALT conventions (e.g., bound and free morphemes were segmented, unintelligible portions of speech were indicated by ' $X X$ '). Narrative audio files were initially transcribed by a primary transcriber, and then a secondary transcriber listened to the audio file and reviewed the first draft, providing feedback to the primary transcriber who made the final decision on the 
transcript. Inter-transcriber agreement was calculated for 13 narrative transcripts (4 DS, 4 FXS, 5 TD), which was $19 \%$ of the current sample. The average agreement was $83 \%$ across relevant dimensions of the transcription process: namely, agreement on identification of (a) C-unit boundaries, (b) partly or completely intelligible C-units, (c) complete C-units (i.e., as opposed to abandoned or interrupted C-units), (d) C-units containing mazes, (e) the number of morphemes per C-unit, (f) the number of words per C-unit, (g) the exact lexical and morphemic content of each C-unit, and (h) C-unit type (question, statement, exclamation). Not surprisingly, given the problems in articulation, voice quality, and speaking rate characteristic of DS and FXS (Barnes et al., 2009; Zajac, Harris, Roberts, \& Martin, 2009), inter-transcriber agreement was lower for these two groups ( $81 \%$ and $80 \%$, respectively) than for the TD participants (86\%).

Coding inference use: Modified from the work of Tompkins et al. (2013), we developed a coding scheme to capture instances of inferential language in participants' narratives. We defined inferences as words, phrases, or sentences that went beyond the literal description illustrated in the pictures on the page and were clearly related to the participant's narrative. Specifically, we defined 15 types of inferences across five primary categories (see Table 2). Character actions/attempts included any mention of physical actions carried out or attempted by a character that were not directly visible on the page spread. Internal state references included mental states as well as other internal attributes of the characters (e.g., personality traits). References to causality explicitly connected two causally related events or referenced one event as the direct result of another event. Use of character dialogue included any instances when the participant gave a character a voice, either by speaking in first person or stating an internal dialogue. Other inferences included general inferences that went beyond what was directly visible on the page spread and did not fall into one of the abovementioned categories (e.g., negation; character role).

Inferences were coded at the utterance level by inference type; that is, each inference type was assigned a unique code so that a single utterance could be coded for the presence of more than one inference type. Utterances that were abandoned or interrupted were not coded for inferences; however, utterances containing partially unintelligible portions were still coded if there was a clear inference produced within the intelligible portion of the utterance. Verbatim utterance repetitions that were produced on the same page spread were assigned any given code only once, upon first mention.

One trained graduate student and one trained undergraduate student applied the coding scheme described above at the utterance level to all narrative transcripts. Approximately $20 \%$ of the narrative transcripts were coded independently by both coders to assess point-bypoint inter-coder agreement, which averaged $91 \%$ across participant groups (range: 71$100 \%$ ). The low end of the range (i.e., $71 \%$ agreement) was represented by one transcript for which intelligibility was particularly poor, complicating coding decisions; for this particular participant, the two coders reconciled differences and made a consensus decision. We used SALT to compute the proportion of utterances containing each type of inference as well as the proportion of utterances containing any instance of inferential language; these proportion scores were used in all reported analyses. 
Mean length of utterance (MLU): We computed participants' MLU in morphemes from their narrative transcripts using SALT software. Only complete and intelligible C-units were included.

Lexical diversity: We used SALT to compute the number of different word roots produced in the first $50 \mathrm{C}$-units of each participant's narrative language sample. If a participant produced fewer than $50 \mathrm{C}$-units, we calculated lexical diversity from the entire sample. Higher lexical diversity scores reflect greater expressive vocabulary. We used these scores to further describe the expressive language abilities of our participants and in exploratory analyses.

Total number of C-units: We used SALT software to compute the total number of C-units produced during each participant's narrative to further describe our participant language samples.

\section{Leiter International Performance Scale-Revised (Leiter-R; Roid \& Miller, 1997)}

-The Leiter-R, normed for ages 2-21 years, is a standardized measure of nonverbal intelligence frequently used in populations with intellectual and developmental disabilities because it is nonverbal in administration and in response method. In the current study, we used the Brief IQ Screener, which is comprised of four subtests: Figure Ground, Form Completion, Repeated Patterns, and Sequential Order. We used composite growth scores to match participant groups by nonverbal cognitive ability level because growth scores reflect raw ability level on an equal interval scale that accounts for varying difficulty across items. For ease of interpretation, we also report standard scores, although it should be noted that $30 \%$ of participants with DS $(n=7)$ and $23 \%$ of participants with FXS $(n=5)$ scored at the floor level Leiter-R Brief IQ standard score.

\section{Results}

\section{Analytic Approach}

To address Aim 1, we conducted an analysis of variance (ANOVA) to examine the impact of participant group on overall inferential language use. Then, we conducted an analysis of covariance (ANCOVA) to examine whether potential group differences changed after accounting for the effect of MLU (Aim 3). Based on the initial findings reported below, in keeping with the focus of the current study on DS, and importantly, to limit the number of statistical comparisons, we proceeded to address Aim 2 (inference subtypes) with a more focused approach.

Specifically, we utilized nested regression to examine the impact of participant group on inferential language use by subtype. We dummy coded variables for participant group into binary variables, using DS as the reference group to reflect the focus on DS in the current study. The "TD" binary variable represented the TD-DS group comparison, and the "FXS" binary variable represented the FXS-DS group difference. We used stepwise regression to examine potential group differences after controlling for the effect of MLU on inferential language use. Thus, in Step 1 we entered the "TD" and "FXS" binary variables representing the group comparisons, and in Step 2 we entered MLU. 


\section{Group Differences in Overall Inferential Language Use}

See Table 3 for proportional inferential language use by participant group. We first examined whether there were group differences in the proportion of utterances containing any type of inferential language. This ANOVA revealed a significant effect of group, $F(2,65)=4.676, p$ $=.013, \eta_{p}{ }^{2}=.126$. LSD pairwise comparisons showed that the group with DS used proportionately less inferential language than either the group with FXS $(p=.031)$ or TD $(p$ $=.005)$, with no significant difference between the groups with FXS and TD $(p=.496)$.

In the second model, an ANCOVA including MLU as a covariate again revealed a significant effect of group, $F(2,64)=5.405, p=.007, \eta_{p}{ }^{2}=.145$, in addition to a significant effect of the covariate, $F(1,64)=88.375, p<.001, \eta_{p}^{2}=.580$. LSD pairwise comparisons showed that, with the inclusion of MLU, there was no longer a significant difference in performance between the groups with DS and TD $(p=.099)$; interestingly, however, the difference between the groups with DS and FXS remained significant $(p=.002)$, again with the participants with FXS showing greater inferential language use than those with DS. The difference between the groups with FXS and TD remained below the level of statistical significance $(p=.138)$.

\section{Group Differences by Inference Type}

See Table 3 for the proportional use of different types of inferential language by participant group. Because use of the different types of inferential language did not all correlate well with each other, the assumptions of multivariate modeling were not met. Therefore, to address Aim 2, we ran five separate models, one for each category of inference type. See the 'Analytic Approach' section above for a description of the dummy coding procedures and analyses utilized below.

Character actions/attempts-In the first step of our stepwise regression model, the effect of participant group was significant, $F(2,65)=3.233, p=.046, R^{2}=.090$, accounting for $9 \%$ of the variance in inferential language use. An examination of the standardized coefficients revealed that only the TD-DS $(\beta=.335, p=.016)$ group difference was significant (FXS-DS $\beta=.240, p=.083$ ), with the TD group outperforming the group with DS.

In the second step, the inclusion of MLU resulted in a statistically significant change to the model, $R^{2}$ change $=.427, p<.001$, with MLU accounting for $43 \%$ of unique variance. The full model was also significant, $F(3,64)=22.837, p<.001, R^{2}=.517$, indicating that the combination of participant group and MLU accounted for $52 \%$ of the total variance in inferential language referring to character actions/attempts. Further examination of the standardized coefficients revealed that, with the inclusion of MLU, the TD-DS group difference was no longer significant ( $\beta=.116, p=.268$ ); interestingly, however, the FXS-DS group difference became significant after accounting for $\operatorname{MLU}(\beta=.232, p=.024)$, showing that the group with FXS outperformed the group with DS.

Internal state references-In the first step, the effect of participant group was only marginally significant, $F(2,65)=3.104, p=.052, R^{2}=.087$, accounting for $9 \%$ of the 
variance. The standardized coefficients revealed that, again, only the TD-DS group difference $(\beta=.339, p=.015$ ) was significant (FXS-DS $\beta=.186, p=.178$ ), again with the TD participants outperforming those with DS.

In the second step, the inclusion of MLU resulted in a statistically significant change to the overall model, $R^{2}$ change $=.183, p<.001$, with MLU accounting for $18 \%$ of unique variance. The full model was also significant, $F(3,64)=7.895, p<.001, R^{2}=.270$, indicating that the participant group and MLU combination accounted for $27 \%$ of the total variance in inferential language referring to character internal states. After the inclusion of MLU, however, neither group difference remained significant (TD-DS $\beta=.196, p=.131$; FXS-DS $\beta=.180, p=.147$ ).

Causal references-Because there was a low rate of causal referencing across the participant groups, with many participants not including any references to causality, we converted participant scores into a dichotomous variable (no causal referencing vs. some causal referencing). We performed a two-predictor logistic regression analysis to examine the relationship between the likelihood that a participant used causal language depending on his or her diagnostic group (using the binary group variables) and MLU.

A test of the full model against a constant only model was significant $\left(\chi^{2}[3]=23.415, p<\right.$. 001), indicating that the model including participant group and MLU as predictors was a better fit than the null model containing no predictors. The Cox and Snell (.291) and Nagelkerke (.431) values (i.e., variations of $R^{2}$ ) indicated that the full model was 29-43\% predictive. Prediction success overall was $82 \%$ (96\% for 'no causal referencing' and $41 \%$ for 'some causal referencing'). An examination of the individual predictor coefficients revealed that only MLU was significant (Wald criterion $=12.033, p=.001$ ). The participant group predictors were not significant (TD-DS Wald criterion $=0.194, p=.659$; FXS-DS Wald criterion $=1.037, p=.309$ ).

Character dialogue-In the first step, the effect of participant group was not significant, $F(2,65)=1.683, p=.194, R^{2}=.049$, accounting for only $5 \%$ of the variance. In the second step, the inclusion of MLU resulted in a statistically significant change to the overall model, $R^{2}$ change $=.083, p=.016$, resulting in the full model becoming significant, $F(3,64)=$ $3.256, p=.027, R^{2}=.132$. Here, the full model accounted for $13 \%$ of the total variance in inferential language as character dialogue. After including MLU, the group differences were still not significant (TD-DS $\beta=-.095, p=.499$; FXS-DS $\beta=.219, p=.106$ ).

Other inferential language-In the first step, the effect of participant group was significant, $F(2,65)=3.256, p=.045, R^{2}=.091$, and accounted for $9 \%$ of the variance. Only the FXS-DS group difference was significant (FXS-DS $\beta=.344, p=.014$; TD-DS $\beta=.209$, $p=.130$ ), revealing that the group with FXS used more other types of inferential language than the group with DS.

In the second step, the inclusion of MLU resulted in a statistically significant change to the overall model, $R^{2}$ change $=.386, p<.001$, with MLU explaining $39 \%$ of unique variance. The full model was also significant, $F(3,64)=19.470, p<.001, R^{2}=.477$; the combination 
of participant group and MLU accounted for $48 \%$ of the total variance in other inferential language. After the inclusion of MLU, the FXS-DS comparison remained significant $(\beta=$. $337, p=.002)$, and the TD-DS comparison was still not significant $(\beta<.001, p=.997)$.

\section{Exploratory Analyses}

To further contextualize our results, we conducted several exploratory analyses and report the general findings here. First, we re-ran all analyses covarying a different metric of language, lexical diversity, to represent expressive vocabulary or semantic ability, as semantic ability is also important to inferential language. Overall, the pattern of results remained unchanged with the inclusion of lexical diversity as a covariate, and total variance explained in each model was less than when MLU was included as the covariate. Thus, our results are stronger with the inclusion of MLU as a proxy for expressive language ability ${ }^{1}$.

Second, because there were only three females in the sample with FXS, we were unable to broadly consider sex differences in our analyses. However, acknowledging that the genetic mechanisms underlying FXS suggest some phenotypic sex differences, we re-ran all analyses with the three females removed from our FXS sample, to ensure that they did not unduly influence our group findings. The pattern of results did not change with the exclusion of females from the FXS sample.

\section{Discussion}

The purpose of this study was to examine inferential language use during narrative storytelling by children and adolescents with DS relative to same-aged peers with FXS and younger TD children matched by nonverbal cognitive ability level. First, we aimed to compare groups according to their overall rate of inferential language use (i.e., the proportion of utterances containing inferential language). Second, because prior literature led to the hypothesis that inferential language use may vary by inference type, we also compared groups according to their use of each of five categories of inferential language. Finally, we examined whether group differences in inferential language use remained after accounting for the potential influence of MLU (as a metric of expressive morphosyntax).

The individuals with DS used proportionately less inferential language overall in their narratives than those with TD or FXS. This finding suggests specificity to the lack of inferential language use observed in DS, at least relative to those with FXS and intellectual disability, informing our work on the DS phenotype during late childhood and adolescence. After accounting for the variability explained by MLU, however, there was no longer a difference between the groups with DS or TD in inferential language use, suggesting that this group difference was driven by a difference in expressive morphosyntax abilities. Interestingly, though, after accounting for MLU, the difference between the groups with DS and FXS remained significant. That is, individuals with DS used proportionately less inferential language than those with FXS even after MLU was taken into account. This

\footnotetext{
${ }^{1}$ We also ran all analyses using MLU in words rather than MLU in morphemes. The pattern of results was similar, except that the TDDS group difference in overall inferential language remained statistically significant after controlling for MLU in words. The FXS-TD group difference, however, was consistent across all analyses, regardless of covariate used.
} 
suggests that along with expressive morphosyntax, another underlying factor may be driving the lack of inferential language use during narrative storytelling in individuals with DS. Future research should investigate other potential underlying factors, perhaps in the social cognitive domain (e.g., perspective taking).

Importantly, the extant literature on the DS phenotype leads to the hypothesis that individuals with DS may show a differential pattern of inferential language use across subtypes. Thus, our second aim was to examine group differences by type of inferential language used (i.e., the proportion of utterances containing each inference type). The inference subtypes from our coding scheme were supported by the lack of significant correlations among inference types, suggesting more distinct categories. These results also shed light on the group differences observed in overall inferential language use.

Before accounting for MLU, we observed group differences for most inference types. In all cases where group differences were observed, the group with DS used significantly less inferential language than either the TD group (references to character actions/attempts; internal state references) or the group with FXS (other inferential language). Interestingly, our results again changed once MLU was included. As was the case with overall inferential language use, there was no longer a significant difference between participants with DS or TD in references to character actions or attempts; however, a significant difference emerged between the group with DS and the group with FXS for this inference category, such that participants with DS used significantly fewer references to character actions or attempts than did participants with FXS. This finding fits with that of overall inferential language and may mean that the underlying social-cognitive factors driving this impairment in DS are more specific to understanding others' intentions or goals that motivate their actions (e.g., understanding intentionality, see Hahn et al., 2013). Linguistically, the communication of character actions requires the use of certain types of verbs, and there is some evidence that verb use is impaired in DS beyond level of MLU (Channell et al., 2015; Hesketh \& Chapman, 1998). Thus, verb use may be yet another contributing factor and should also be considered in future research.

Although we did not observe significant group differences for the use of causal references or character dialogue, these types of inferential language yielded the lowest rates of use for all participants. Regardless, MLU was still a significant predictor in inferential language use for all categories, including these two. Thus, there is a clear link between complexity of sentence structure and use of inferential language during narration in individuals with DS. Because our study design was cross-sectional, we cannot draw conclusions regarding causality. However, we hypothesize two potential explanations that warrant further examination in future studies.

It may be that the social cognitive ability to draw inferences is impaired in DS, and this impairment precludes the need for using more complex syntax. Alternatively, it may be that the syntactic limitations characteristic of individuals with DS may constrain their use of inferential language during narration, a relatively complex communication context. Both of these potential explanations fit with the current literature on the DS phenotype. The second explanation fits with studies reporting that the characteristically low expressive syntactic 
abilities of children and adolescents with DS may hinder the communication of other kinds of narrative content (Boudreau \& Chapman, 2000; Finestack \& Abbeduto, 2010; Keller-Bell \& Abbeduto, 2007; Miles \& Chapman, 2002). The first explanation fits with DS phenotypic research documenting impairments in more complex social cognitive abilities (Abbeduto et al., 2001; Beeghly \& Cicchetti, 1997; Hahn et al., 2013; Hesketh \& Chapman, 1998; Zelazo et al., 1996). However, due to the scarcity of literature on higher-order social cognitive reasoning in older children or adolescents with DS, less evidence currently supports this explanation, and future research is needed to address this possibility. This is an important consideration for future endeavors, especially given the potential syndrome-specific nature of some of our findings that suggest particular difficulty in some aspects of inferential language beyond what can be attributed to expressive morphosyntax. Future research should also examine whether these syndrome specific differences remain after considering other aspects of expressive language beyond MLU or expressive vocabulary.

Regardless of the causal factors involved, there is a clear need for clinical approaches that support the use of inferential language and complex sentences structures in individuals with DS. Results from our study suggest that interventions aimed at enhancing spoken language in DS should consider targeting inferential language, with a particular focus on discussing character intentions, goal-oriented actions, and internal states, as these aspects of inferential language were found to be impaired in our sample. One potential intervention approach in the context of shared storytelling could include strategies such as asking the child whquestions, which has been found to be effective in eliciting inferential language use and increasing language outcomes in TD children and in those with language impairment (Dunst, Williams, Trivette, Simkus, \& Hamby, 2012). Such strategies have been successfully embedded into parent-implemented interventions to increase language outcomes for youth with FXS (McDuffie et al., 2016). The current study's findings support the need for similar interventions in youth with DS, although our findings specific to DS also suggest the need for some adaptations for this population.

The current study is not without its limitations. First, in considering the applicability of our findings to parent-child shared storytelling contexts, future research should consider accounting for varying exposure to literacy related activities, as this may play an important role in the development of inferential language use. Also, because MLU was derived from the same narrative task in which inferential language was measured, future research would benefit from the inclusion of separate tasks to measure each of these skills. Further, other more detailed metrics of grammatical complexity beyond MLU (e.g., Index of Productive Syntax [Scarborough, 1990]; Developmental Sentence Scoring [Lee, 1974]) may be more sensitive to the structural language difficulties observed in individuals with DS (Finestack \& Abbeduto, 2010; Price, Roberts, Hennon, Berni, Anderson, \& Sideris, 2008) and may elucidate the role of structural language in inferential language use in this population. Also, although the Narrative Task utilized in this study provides ample opportunities to discuss cause and effect, few of the participants in the range of developmental levels included in our study were able to spontaneously produce references to causality. Future research should also focus on prompting individuals for different types of inferring, include causal reasoning, to document this important social cognitive ability. Finally, although our findings suggest some potential syndrome specificity to the DS phenotype regarding inferential 
language use, future research is needed to confirm or reject this suggestion. The inclusion of comparison groups with intellectual disability of other origins beyond FXS as well as those with language impairment without intellectual disability will be important to elucidating the discussion regarding syndrome specificity.

\section{Acknowledgments}

We are most thankful for all the families who participated in this study. We also thank the countless staff who worked with participant recruitment and scheduling, data collection, and language sample transcription. We thank Taylor Hoffman for her contributions to the coding specific to this project. This research was supported by NIH grants R01HD024356 and U54HD079125 (PI: Abbeduto).

\section{References}

Abbeduto L, Benson G, Short K, Dolish J. Effects of sampling context on the expressive language of children and adolescents with mental retardation. Mental Retardation. 1995; 33:279-288. [PubMed: 7476250]

Abbeduto L, Murphy MM, Cawthon SW, Richmond EK, Weissman MD, Karadottir S, O'Brien A. Receptive language skills of adolescents and young adults with Down or fragile X syndrome. American Journal on Mental Retardation. 2003; 108:149-160. [PubMed: 12691594]

Abbeduto L, Murphy MM, Kover ST, Giles ND, Karadottir S, Amman A, ... Nollin KA. Signaling noncomprehension of language: A comparison of fragile $\mathrm{X}$ syndrome and Down syndrome. American Journal on Mental Retardation. 2008; 113:214-230. [PubMed: 18407723]

Abbeduto L, Pavetto M, Kesin E, Weissman M, Karadottir S, O’Brien A, Cawthon S. The linguistic and cognitive profile of Down syndrome: Evidence from a comparison with fragile $\mathrm{X}$ syndrome. Down Syndrome Research and Practice. 2001; 7:9-15. DOI: 10.3104/reports.109

Abbeduto L, Warren SF, Conners FA. Language development in Down syndrome: From the prelinguistic period to the acquisition of literacy. Mental Retardation and Developmental Disabilities Research Reviews. 2007; 13:247-261. DOI: 10.1002/mrdd.20158 [PubMed: 17910087]

Aldrich NJ, Tenenbaum HR, Brooks PJ, Harrison K, Sines J. Perspective taking in children's narratives about jealousy. British Journal of Developmental Psychology. 2011; 29:86-109. DOI: 10.1348/026151010X533238 [PubMed: 21288255]

Bamberg M, Marchman V. What holds a narrative together? The linguistic encoding of episode boundaries. Papers in Pragmatics. 1990; 4:58-121.

Barnes E, Roberts J, Long SH, Martin GE, Berni MC, Mandulak KC, Sideris J. Phonological accuracy and intelligibility in connected speech of boys with fragile $\mathrm{X}$ syndrome or Down syndrome. Journal of Speech, Language, and Hearing Research. 2009; 52:1048-1061. DOI: 10.1044/1092-4388(2009/08-0001)

Baron-Cohen S, Leslie AM, Frith U. Mechanical, behavioural and intentional understanding of picture stories in autistic children. British Journal of Developmental Psychology. 1986; 4:113-125.

Beeghly M, Cicchetti D. Talking about self and other: Emergence of an internal state lexicon in young children with Down syndrome. Development and Psychopathology. 1997; 9:729-748. [PubMed: 9449003]

Berman RA. Narrative competence and storytelling performance: How children tell stories in different contexts. Journal of Narrative and Life History. 1995; 5:285-313.

Berry-Kravis E, Doll E, Sterling A, Kover ST, Schroeder SM, Mathur S, Abbeduto L. Development of an expressive language sampling procedure in fragile X syndrome: A pilot study. Journal of Developmental and Behavioral Pediatrics. 2013; 34:245-251. DOI: 10.1097/dbp. 0b013e31828742fc [PubMed: 23669871]

Boudreau DM, Chapman RS. The relationship between event representation and linguistic skill in narratives of children and adolescents with Down syndrome. Journal of Speech, Language, and Hearing Research. 2000; 43:1146-1159. DOI: 10.1044/jslhr.4305.1146

Cain K, Oakhill JV. Inference making and its relation to comprehension failure. Reading and Writing. 1999; 11:489-503. DOI: 10.1023/A:1008084120205 
Cain, K., Oakhill, J. Reading comprehension development from seven to fourteen years: Implications for assessment. In: Sabatini, JP.Albro, ER., Reilly, T., editors. Measuring up: Advances in how to assess reading ability. New York, NY: Rowman \& Littlefield; 2012. p. 59-76.

Carpendale, J., Lewis, C. How children develop social understanding. Malden, MA: Blackwell Publishing; 2006.

Channell MM, McDuffie AS, Bullard LM, Abbeduto L. Narrative language competence in children and adolescents with Down syndrome. Frontiers in Behavioral Neuroscience. 2015; 9:283-293. DOI: 10.3389/fnbeh.2015.00283 [PubMed: 26578913]

Chapman RS, Hesketh LJ. Behavioral phenotype of individuals with Down syndrome. Mental Retardation and Developmental Disabilities Research Reviews. 2000; 6:84-95. DOI: 10.1002/1098-2779(2000)6:2<84::AID-MRDD2>3.0.CO;2-P [PubMed: 10899801]

Chapman RS, Seung HK, Schwartz SE, Kay-Raining Bird E. Language skills of children and adolescents with Down syndrome: II. Production deficits. Journal of Speech, Language, and Hearing Research. 1998; 41:861-873.

Dunst C, Williams A, Trivette C, Simkus A, Hamby D. Relationships between inferential book reading strategies and young children's language and literacy competence. Center for Early Literacy Learning. 2012; 5:1-10.

Estigarribia B, Martin GE, Roberts JE, Spencer A, Gucwa A, Sideria J. Narrative skill in boys with fragile $X$ syndrome with and without autism spectrum disorder. Applied Psycholinguistics. 2011; 32:359-388. DOI: 10.1017/S0142716410000445 [PubMed: 21516264]

Fidler DJ. The emergence of a syndrome specific personality profile in young children with Down syndrome. Down Syndrome Research and Practice. 2006; 10:53-60. http://dx.doi.org/10.3104/ reprints.305.

Fidler DJ, Most DE, Booth-LaForce C, Kelly JF. Emerging social strengths in young children with Down syndrome. Infants \& Young Children. 2008; 21:207-220. http://dx.doi.org/10.1097/01.IYC. 0000324550.39446.1f.

Finestack LH, Abbeduto L. Expressive language profiles of verbally expressive adolescents and young adults with Down syndrome or fragile X syndrome. Journal of Speech, Language, and Hearing Research. 2010; 53:1334-1348. DOI: 10.1044/1092-4388(2010/09-0125)

Finestack LH, Palmer M, Abbeduto L. Macrostructural narrative language of adolescents and young adults with Down syndrome or fragile X syndrome. American Journal of Speech-Language Pathology. 2012; 21:29-46. DOI: 10.1044/1058-0360(2011/10-0095) [PubMed: 22049405]

Finestack LH, Sterling AM, Abbeduto L. Discriminating Down syndrome and fragile X syndrome based on language ability. Journal of Child Language. 2013; 40:244-265. http://doi.org/10.1017/ S0305000912000207. [PubMed: 23217297]

Hahn LJ, Fidler DJ, Hepburn SL, Rogers SJ. Early intersubjective skills and the understanding of intentionality in young children with Down syndrome. Research in Developmental Disabilities. 2013; 34:4455-4465. DOI: 10.1016/j.ridd.2013.09.027 [PubMed: 24112996]

Hesketh LJ, Chapman RS. Verb use by individuals with Down syndrome. American Journal on Mental Retardation. 1998; 103:288-304. DOI: 10.1352/0895-8017(1998)103<0288:vubiwd>2.0.co;2 [PubMed: 9833659]

Hogan-Brown AL, Losh M, Martin GE, Mueffelmann DJ. An investigation of narrative ability in boys with autism and fragile $\mathrm{X}$ syndrome. American Journal on Intellectual and Developmental Disabilities. 2013; 118:77-94. DOI: 10.1352/1944-7558-118.2.77 [PubMed: 23464607]

Keller-Bell YD, Abbeduto L. Narrative development in adolescents and young adults with fragile X syndrome. American Journal of Mental Retardation. 2007; 4:289-299.

Kendeou P, Bohn-Gettler C, White MJ, van den Broek P. Children's inference generation across different media. Journal of Research in Reading. 2008; 31:259-272. DOI: 10.1111/j. 1467-9817.2008.00370.x

Klusek J, Martin GE, Losh M. A comparison of pragmatic language in boys with autism and fragile X syndrome. Journal of Speech, Language, and Hearing Research. 2014; 57:1692-1707. DOI: 10.1044/2014_JSLHR-L-13-0064 
Kover ST, McDuffie A, Abbeduto L, Brown WT. Effects of sampling context on spontaneous expressive language in males with fragile X syndrome or Down syndrome. Journal of Speech, Language, and Hearing Research. 2012; 55:1022-1038. DOI: 10.1044/1092-4388(2011/11-0075)

Lee, L. Developmental sentence analysis. Evanston, IL: Northwestern University Press; 1974.

Lee M, Bush L, Martin GE, Barstein J, Maltman N, Klusek J, Losh M. A multi-method investigation of pragmatic development in individuals with Down syndrome. American Journal on Intellectual and Developmental Disabilities. 2017; 122:189-309. DOI: 10.1352/1944-7558-122.4.289

Loban, W. Language development: Kindergarten through grade twelve. Urbana, IL: National Council of Teachers of English; 1976.

Losh M, Martin GE, Klusek J, Hogan-Brown AL, Sideris J. Social communication and theory of mind in boys with autism and fragile X syndrome. Frontiers in Psychology. 2012; 3:1-12. DOI: 10.3389/fpsyg.2012.00266 [PubMed: 22279440]

Martin GE, Losh M, Estigarribia B, Sideris J, Roberts J. Longitudinal profiles of expressive vocabulary, syntax, and pragmatic language in boys with fragile X syndrome or Down syndrome. International Journal of Language \& Communication Disorders. 2013; 48:432-443. DOI: 10.1111/1460-6984.12019 [PubMed: 23889838]

Martin GE, Roberts JE, Helm-Estabrooks N, Sideris J, Vanderbilt J, Moskowitz L. Perseveration in the connected speech of boys with fragile $\mathrm{X}$ syndrome with and without autism spectrum disorder. American Journal on Intellectual and Developmental Disabilities. 2012; 117:384-339. DOI: 10.1352/1944-7558-117.5.384 [PubMed: 22998486]

McCabe A, Peterson C. What makes a narrative memorable? Applied Psycholinguistics. 1990; 11:7382.

McDuffie AM, Abbeduto L, Lewis P, Kover ST, Kim JS, Weber A, Brown WT. Autism spectrum disorder in children and adolescents with fragile $\mathrm{X}$ syndrome: Within-syndrome differences and age-related changes. American Journal on Intellectual and Developmental Disabilities. 2010; 115:307-326. [PubMed: 20567604]

McDuffie A, Machalicek W, Bullard L, Nelson S, Mello M, Tempero-Feigles R, ... Abbeduto L. A spoken-language intervention for school-aged boys with fragile $\mathrm{X}$ syndrome. American Journal on Intellectual and Developmental Disabilities. 2016; 121:236-265. DOI: 10.1352/1944-7558-121.3.236 [PubMed: 27119214]

McDuffie, A., Thurman, AJ., Channell, MM., Abbeduto, L. Language disorders in children with intellectual disability of genetic origin. In: Schwartz, R., editor. Handbook of Child Language Disorders. 2. New York: Taylor \& Francis; 2017. p. 52-81.

Miles S, Chapman RS. Narrative content as described by individuals with Down syndrome and typically developing children. Journal of Speech, Language, and Hearing Research. 2002; 45:175189. DOI: $10.1044 / 1092-4388(2002 / 013)$

Miller, J. Profiles of language development in children with Down syndrome. In: Miller, J.Leddy, M., Leavitt, L., editors. Improving the communication of people with Down syndrome. Baltimore: Paul H. Brookes; 1999. p. 11-40.

Miller, J., Iglesias, A. Systematic Analysis of Language Transcripts (SALT Version 9) [Computer Software]. Madison, WI: Language Analysis Lab, University of Wisconsin-Madison; 2006.

Moore DG, Oates JM, Hobson RP, Goodwin J. Cognitive and social factors in the development of infants with Down syndrome. Down Syndrome Research and Practice. 2002; 8(2):43-52. http:// dx.doi.org/10.3104/reviews.129.

Phillips BA, Loveall SJ, Channell MM, Conners FA. Matching variables for research involving youth with Down syndrome: Leiter-R versus PPVT-4. Research in Developmental Disabilities. 2014; 35:429-438. [PubMed: 24361811]

Presson AP, Partyka G, Jensen KM, Devine OJ, Rasmussen SA, McCabe LL, McCabe ERB. Current estimate of Down syndrome population prevalence in the United States. The Journal of Pediatrics. 2013; 163:1163-1168. [PubMed: 23885965]

Price J, Roberts J, Hennon EA, Berni MC, Anderson KL, Sideris J. Syntactic complexity during conversation of boys with fragile X syndrome and Down syndrome. Journal of Speech, Language, and Hearing Research. 2008; 51:3-15. 
Roberts J, Martin GE, Moskowitz L, Harris AA, Foreman J, Nelson L. Discourse skills of boys with fragile X syndrome in comparison to boys with Down syndrome. Journal of Speech, Language, and Hearing Research. 2007; 50:475-492. doi:1092-4388/07/5002-0475.

Roid, G., Miller, L. Leiter International Performance Scale-Revised. Wood Dale, IL: Stoelting; 1997.

Scarborough HS. Index of productive syntax. Applied Psycholinguistics. 1990; 11:1-22.

Simon JA, Keenan JM, Pennington BF, Taylor AK, Hagerman RJ. Discourse processing in women with fragile X syndrome: Evidence for a deficit establishing coherence. Cognitive Neuropsychology. 2001; 18:1-18. [PubMed: 20945204]

Smith E, Naess KB, Jarrold C. Assessing pragmatic communication in children with Down syndrome. Journal of Communication Disorders. 2017; 68:10-23. http://dx.doi.org/10.1016/j.jcomdis. 2017.06.003. [PubMed: 28624322]

Sudhalter V, Belser RC. Conversational characteristics of children with fragile X syndrome: Tangential language. American Journal on Mental Retardation. 2001; 106:389-400. [PubMed: 11531459]

Symons D. Mental state discourse, theory of mind, and the internalization of self-other understanding. Developmental Review. 2004; 24:159-188.

Tager-Flusberg H. Autistic children's talk about psychological states: Deficits in the early application of a theory of mind. Child Development. 1992; 63:161-172. [PubMed: 1551324]

Tager-Flusberg H, Anderson M. The development of contingent discourse ability in autistic children. Journal of Child Psychology and Psychiatry. 1991; 52:1123-1134.

Tompkins V, Guo Y, Justice LM. Inference generation, story comprehension, and language skills in the preschool years. Reading and Writing. 2013; 26:403-429. DOI: 10.1007/s11145-012-9374-7

Trabasso T, Stein NL, Rodkin PC, Munger MP, Baughn CR. Knowledge of goals and plans in the online narration of events. Cognitive Development. 1992; 7:133-170.

Wenner JA. Preschoolers' comprehension of goal structure in narratives. Memory. 2004; 12:193-202. DOI: 10.1080/09658210244000478 [PubMed: 15250184]

Zajac DJ, Harris AA, Roberts JE, Martin GE. Direct magnitude estimation of articulation rate in boys with fragile X syndrome. Journal of Speech, Language, and Hearing Research. 2009; 52:1370 1379. DOI: 10.1044/1092-4388(2009/07-0208)

Zelazo PD, Burrack JA, Benedetto E, Frye D. Theory of mind and rule use in individuals with Down's syndrome: A test of uniqueness and specificity claims. Journal of Child Psychology and Psychiatry. 1996; 37:479-484. [PubMed: 8735448] 


\section{Highlights}

- Youth with DS used less inferential language than the other participant groups.

- Inferential language may be unique to the DS phenotype, at least relative to FXS.

- $\quad$ Results varied by inference subtype.

- $\quad$ Expressive morphosyntactic abilities contributed to the observed group differences. 


\section{What this paper adds?}

To date, no studies have comprehensively examined inferential language skill in individuals with Down syndrome (DS). Because inferential language serves as a framework for positive social interactions and learning in academic contexts, it is important to determine whether the phenotypic characteristics of DS impact this important foundation for development. The purpose of the current study, therefore, was to investigate inferential language use by youth with DS in the context of narrative storytelling relative to youth with fragile X syndrome (FXS) and typically developing (TD) children. Comparing participants with DS to younger TD children of similar nonverbal cognitive ability level allowed us to determine whether inferential language is a strength or weakness relative to cognitive developmental level. Including a same-age comparison group of FXS — another cause of intellectual disability — in addition to the TD group provided some insight into the syndrome-specificity of the findings (i.e., the extent to which any differences observed can be attributed to intellectual disability in general or specific phenotypes-DS or FXS—within intellectual disability). Finally, we examined the contribution of expressive morphosyntactic ability to group differences observed in inferential language use. 


\section{Table 1}

Descriptive Characteristics of Participants

\begin{tabular}{|l|c|c|c|}
\hline & DS $(\boldsymbol{n}=\mathbf{2 3})$ & FXS $(\boldsymbol{n}=\mathbf{2 2})$ & TD $(\boldsymbol{n}=\mathbf{2 3})$ \\
\hline & Mean (SD) Range & Mean (SD) Range & Mean (SD) Range \\
\hline Chronological age & $\begin{array}{c}12.80(1.59) \\
10.28-15.54\end{array}$ & $\begin{array}{c}12.33(1.74) \\
10.18-16.01\end{array}$ & $\begin{array}{c}4.48(0.86) \\
3.11-6.19\end{array}$ \\
\hline Leiter-R Brief IQ growth scores & $462.09(7.66)$ & $462.82(7.09)$ & $462.22(7.58)$ \\
& $442-474$ & $446-476$ & $442-474$ \\
\hline Leiter-R Brief IQ standard scores ${ }^{a}$ & $42.48(7.07)$ & $44.41(7.87)$ & $110.96(15.50)$ \\
& $36-65$ & $36-65$ & $87-159$ \\
\hline MLU in morphemes & $5.07(2.00)$ & $5.11(1.42)$ & $6.19(1.32)$ \\
& $1.40-9.17$ & $2.83-7.37$ & $4.07-8.83$ \\
\hline Lexical diversity & $67.17(29.94)$ & $65.50(21.02)$ & $84.96(26.45)$ \\
& $15-117$ & $30-107$ & $40-153$ \\
\hline Total number of C-units & $47.09(25.76)$ & $38.18(15.59)$ & $49.04(25.25)$ \\
& $16-139$ & $19-82$ & $25-134$ \\
\hline
\end{tabular}

${ }^{a}$ DS: $n=7$, FXS: $n=5$ scored at the floor standard score of 36 

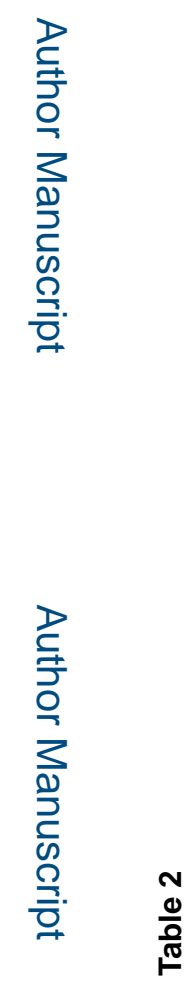

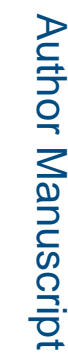

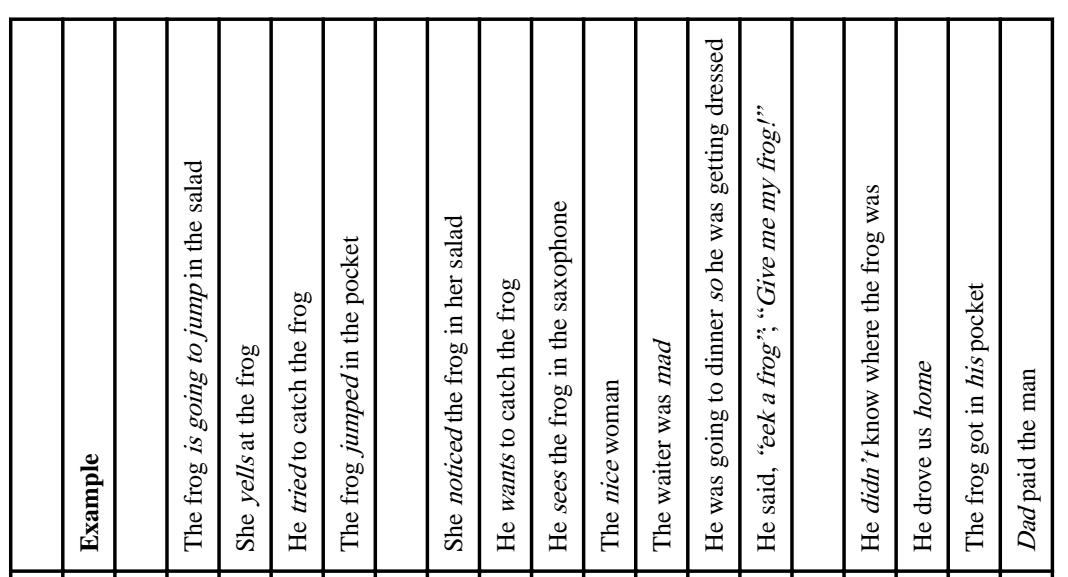

로을

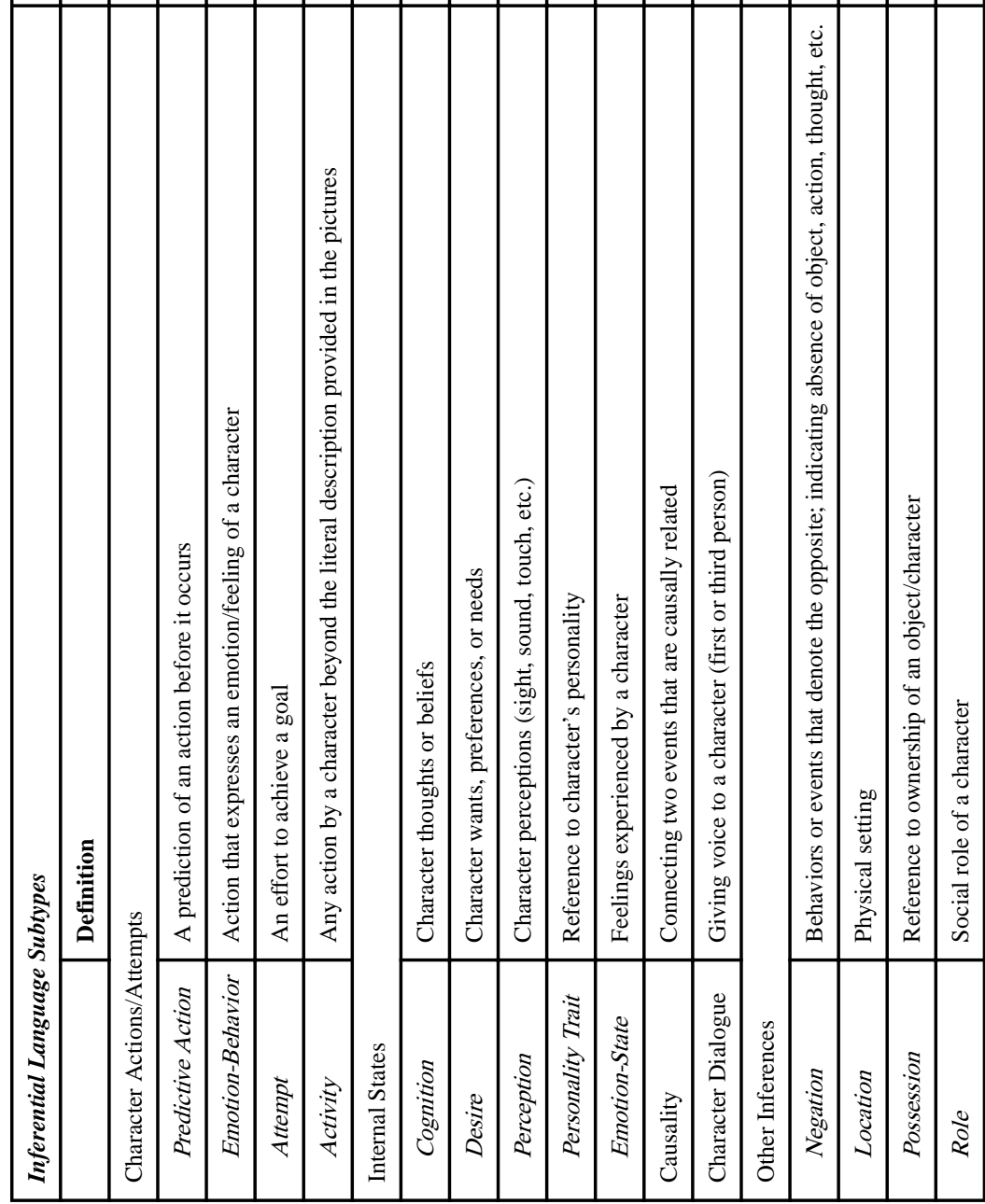

Res Dev Disabil. Author manuscript; available in PMC 2018 December 01. 
Table 3

Proportion of Utterances Containing Each Type of Inferential Language

\begin{tabular}{|l|c|c|c|}
\hline & DS $(\boldsymbol{n = 2 3})$ & FXS $(\boldsymbol{n}=\mathbf{2 2})$ & TD $(\boldsymbol{n}=\mathbf{2 3})$ \\
\hline & Mean (SD) Range & Mean (SD) Range & Mean (SD) Range \\
\hline Overall Inferential Language & $.50(.25)$ & $.65(.21)$ & $.69(.20)$ \\
& $.00-.95$ & $.35-1.00$ & $.34-.96$ \\
\hline Character Actions/Attempts & $.37(.23)$ & $.47(.16)$ & $.51(.20)$ \\
& $.00-.88$ & $.17-.73$ & $.15-.87$ \\
\hline Internal States & $.08(.07)$ & $.11(.09)$ & $.14(.09)$ \\
& $.00-.24$ & $.00-.33$ & $.00-.37$ \\
\hline Causality ${ }^{a}$ & $.02(.04)$ & $.01(.02)$ & $.01(.03)$ \\
& $.00-.18$ & $.00-.07$ & $.00-.10$ \\
\hline Character Dialogue & $.05(.06)$ & $.09(.10)$ & $.05(.07)$ \\
& $.00-.22$ & $.00-.31$ & $.00-.25$ \\
\hline Other Inferences & $.13(.07)$ & $.19(.12)$ & $.17(.07)$ \\
& $.00-.24$ & $.00-.52$ & $.06-.29$ \\
\hline
\end{tabular}

${ }^{a}$ Percentage of each group that included some references to causality: DS $=26 \%, \mathrm{FXS}=14 \%, \mathrm{TD}=35 \%$. 